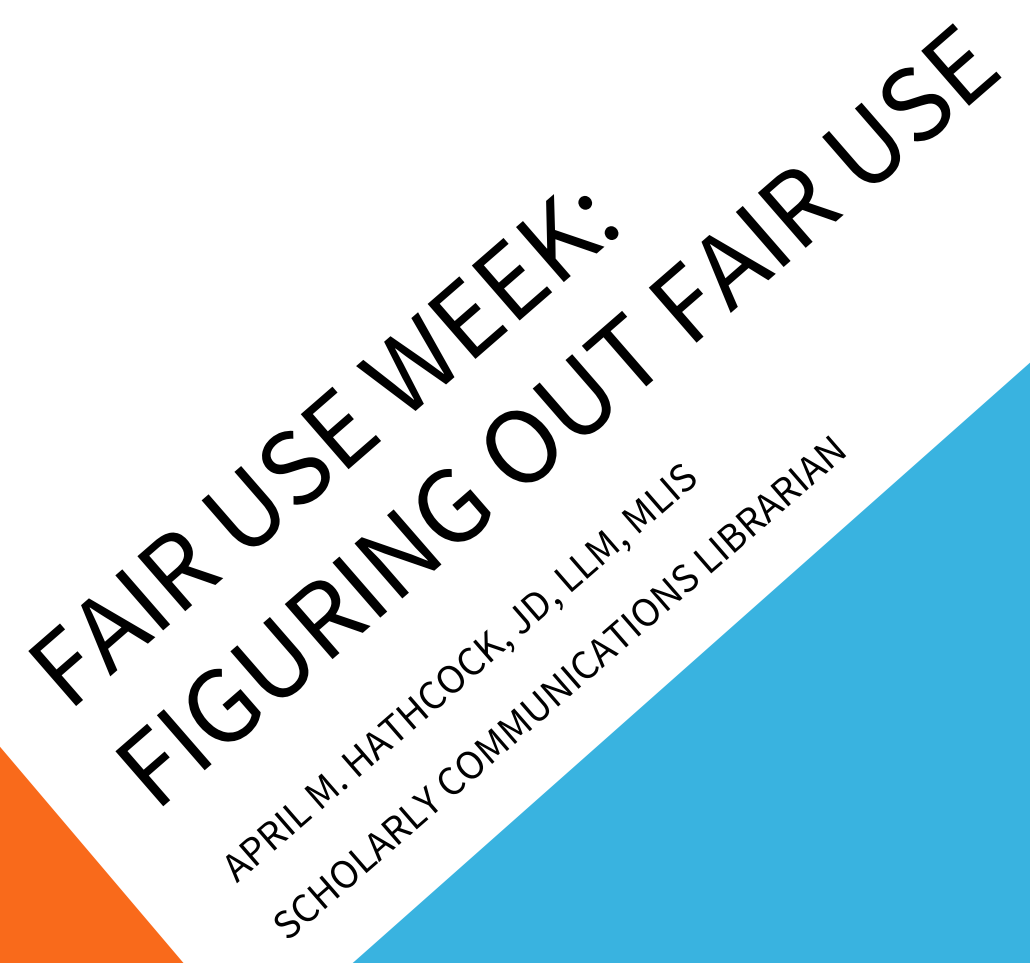

(c) (1) (9) 


\section{COPYRIGHT BASICS}

- Any tangible form of expression

- Attaches automatically

- Regardless of publication or access $\rightarrow$ Material on the internet IS covered by copyright

- Fair use provides an exception to copyright exclusions

\section{guides.nyu.edu/fairuse}

N.B. We're discussing fair use of copyrighted material that is not subject to a license. Licenses always prevail over fair use. 


\section{SECTION 107}

Notwithstanding the provisions of sections 106 and 106A, the fair use of a copyrighted work, including such use by reproduction in copies or phonorecords or by any other means specified by that section, for purposes such as criticism, comment, news reporting, teaching (including multiple copies for classroom use), scholarship, or research, is not an infringement of copyright. In determining whether the use made of a work in any particular case is a fair use the factors to be considered shall include-

(1) the purpose and character of the use, including whether such use is of a commercial nature or is for nonprofit educational purposes;

(2) the nature of the copyrighted work;

(3) the amount and substantiality of the portion used in relation to the copyrighted work as a whole; and

(4) the effect of the use upon the potential market for or value of the copyrighted work.

The fact that a work is unpublished shall not itself bar a finding of fair use if such finding is made upon consideration of all the above factors. 
Fair use. .

is a

right, is a

defense, is

flexible,

and benefits 


\section{FAIR USE: FOUR FACTORS}

What are you using it for?

- Commentary and criticism > Entertainment

- Transformative use > Original use

What are you using?

- Nonfictional, instructional > Fictional, highly creative

How much are you using? How far are you spreading it?

- Excerpts > Entire works

- Password-protected sites > Open web

How much would it cost?

- Not readily available for license > Available for educational license 


\section{FAIR USE FOR IMAGES}

Favors Fair Use

- Medium- to low-res

- Thumbnail, reduced size

- Accompanied by contextual text for criticism/commentary

- Pedagogical/scholarly purpose

\section{Disfavors Fair Use}

- High-res

- Original size

- No contextual text for criticism/commentary

- Entertainment/design purpose 
It can be tricky to determine the creator of a web image. To make giving credit easy, look for images that give you enough information to attribute them.

Be sure to credit images properly.

Crediting images does not guarantee fair use but is still an important part of scholarly practice.

guides.nyu.edu/copyright/images
At minimum, do your best to:

1. Link to back to the original work

2. Give credit to the image creator

3. Follow attribution instructions provided by the source

\section{Generic Image Credit Format:}

Title by A. Creator, via source (CC License Type).

\section{Examples:}

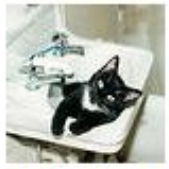

Image credit: Jack in Sink (again) by J. Smith, via Flickr.com (CC NC-BY 2.0).

Image credit: 13694 by A. Mills, via CDC.

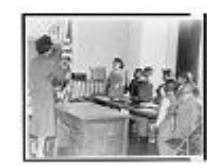

Image credit: Mrs. Claire... by D. DeMarisco, via Library of Congress, Prints \& Photographs Division, NYWT\&S Collection. 


\section{FAIR USE FOR AUDIO/VIDEO}

\section{Favors Fair Use}

- Clips and excerpts

- Accompanied by contextual text for criticism/commentary

- Pedagogical/scholarly purpose

- Made available via streaming

- Behind password protection

- Out of distribution, difficult to locate/purchase

\section{Disfavors Fair Use}

- Full content

- No contextual text for criticism/commentary

- Entertainment purpose

- Made available for download

- Posted on the open web

- Currently in distribution, readily available for licensing 


\section{FAIR USE PRACTICE}

- Posting the scans of 3 short chapters of a book into a password-protected course management system for students to access?

- Ripping a full-length blockbuster film into a password-protected streaming system so your friends and family can enjoy it?

- Ripping a full-length blockbuster film into a password-protected streaming system so your students can study it at home for scholarly critique?

- Making the full-text of a journal article, the rights to which you do not own, available for download on your public website?

- Posting clips of a sound recording on your scholarly website so you can discuss the nuances of musicology?

- Embedding a YouTube video of a dancing kitten with mittens onto your blog because ... dancing kitten with mittens? 


\section{RESOURCES}

NYU Policy on Educational and Research Uses of Copyrighted Materials

- http://www.nyu.edu/about/policies-guidelines-compliance/policies-and-guidelin es/educational-and-research-uses-of-copyrighted-materials-policy-st.html

Copyright Basics Research Guide

- www.guides.nyu.edu/copyright

Copyright for Authors and Creators

- www.guides.nyu.edu/copyrightforauthors

Fair Use Listserv

- FairUse@nyu.edu

Scholarly Communications Librarian

- April Hathcock, 1M-01 Mezzanine, x26258, april.hathcock@nyu.edu 